\title{
Refractive index sensing of gases based on a one-dimensional photonic crystal nanocavity
}

\author{
M. Mohebbi \\ Department of Electrical Engineering, Islamic Azad University, Daneshgah Ave., Qazvin, Iran \\ Correspondence to: M. Mohebbi (mohebbi@qiau.ac.ir)
}

Received: 15 January 2015 - Revised: 26 April 2015 - Accepted: 12 May 2015 - Published: 4 June 2015

\begin{abstract}
Silicon photonic crystal sensors have become very attractive for various optical sensing applications. Using silicon as a material platform provides the ability to fabricate sensors with other photonic devices on a single chip. In this paper, a new optical sensor based on optical resonance in a one-dimensional silicon photonic crystal with an air defect is theoretically studied for refractive index sensing in the infrared wavelength region. The air defect introduces a cavity into the photonic crystal, making it suitable for probing the properties of a gas found within the cavity. This photonic crystal nanocavity is designed to oscillate at a single mode with a high quality factor, allowing for refractive index sensing of gases with a high sensitivity. A method is presented to maximize the sensitivity of the sensor and to obtain a very narrow bandwidth cavity mode for good sensor resolution. We change the thickness of the air layers linearly in the photonic crystals on both sides of the nanocavity and show that a sensitivity of $1200 \mathrm{~nm} \mathrm{RIU}^{-1}$ can be achieved. We present a detailed analysis of the sensor and variations of the layer thicknesses, the cavity length, and the number of periodic layers in the photonic crystal are investigated. This optical sensor has a much simpler design and higher sensitivity compared to other photonic crystal sensors reported previously.
\end{abstract}

\section{Introduction}

Optical sensors are used for a variety of applications in science, technology, and the environment. Most of these optical sensors measure the variations of optical properties as a result of a change in the refractive index of a gas or a liquid. Various structures have been investigated as optical sensors such as fiber Bragg gratings (Fang et al., 2010), step-index fibers and photonic crystal fibers (Lee et al., 2011; Udd and Spillman, 2011), fiber-assisted surface plasmon resonances (Ma et al., 2009), and photonic crystals (Liu and Salemink, 2012; Jágerská et al., 2010; Kang et al., 2010; Bougriou et al., 2013). Recently, optical resonance in photonic crystals has received increased attention for refractive index sensing applications.

Photonic crystals are periodic structures that exhibit a band gap for a range of wavelengths. Photonic crystals with oneand two-dimensional (1-D, 2-D) band gaps have been studied for a variety of refractive index sensing applications. Below, we give some of the most recent and important examples. A new design based on a 2-D photonic crystal slab with a tri- angular lattice pattern has been used for optofluidic sensing with a sensitivity of $636 \mathrm{~nm} \mathrm{RIU}^{-1}$ (Bougriou et al., 2013). A 2-D photonic crystal L7 cavity has been studied for refractive sensing of water and ethanol solutions (Liu and Salemink, 2012). In this design, a sensitivity of $460 \mathrm{~nm} \mathrm{RIU}^{-1}$ has been achieved compared to a sensitivity of about $100 \mathrm{~nm} \mathrm{RIU}{ }^{-1}$ for $\mathrm{L} 3$ cavities. When gases are used as a sensing material, optical sensors must be designed in such a way that the optical field is strong in the free space region. One way is to use an air-slot cavity inside the line defect region of a 2-D photonic crystal. In one method, a cavity is created by reducing the width of the air slot and keeping the photonic crystal lattice unchanged (Jágerská et al., 2010). Using this method, a sensitivity of $510 \mathrm{~nm} \mathrm{RIU}^{-1}$ has been achieved for refractive index sensing of gases in the infrared (IR).

Although these 2-D photonic crystal optical sensors can be used for refractive sensing of liquids and gases, they all have limitations. The fabrication of these sensors is difficult and complicated and the sensitivities that can be achieved by these optical sensors are not high. Because of easy fabrica- 
tion, 1-D photonic crystals have attracted a lot of attention for fabrication of different components in silicon-based photonic integrated circuits. Techniques for fabrication of 1-D dielectric photonic crystal at IR wavelengths are mature and well developed. It is now possible to fabricate 1-D photonic crystals with layer thicknesses reaching the nanometer scale. 1D dielectric multilayer structures, or photonic crystals, have been used in many photonic devices as filters (Vangala et al., 2014), microcavities (Chen et al., 2014b) and optical sensors (Sreekantha et al., 2013; Chen et al., 2014a; Frascella et al., 2013; Nunes et al., 2010).

Several 1-D photonic crystal optical sensors have been suggested for refractive index sensing. A biosensor configuration based on the excitation of surface electromagnetic waves in a 1-D photonic crystal has been proposed (Sreekantha et al., 2013). They show that the sensitivity of the proposed configuration is much higher compared to the sensitivity of conventional surface plasmon resonance biosensors. A refractive index sensor consisting of a 1-D photonic crystal embedded in a microfluidic channel has been investigated, yielding a sensitivity of $836 \mathrm{~nm} \mathrm{RIU}^{-1}$ for several ethanol solutions (Nunes et al., 2010). A 1-D photonic crystal has been used as an optical transducer for biosensing upon the coupling of Bloch surface waves (Frascella et al., 2013). In this design, the photonic structure exhibits an emissive behavior and the fluorescence shift is used as a means for detecting refractive index variations occurring at the 1-D photonic crystal surface. Recently, a terahertz gas sensor using a 1-D photonic crystal cavity has been reported (Chen et al., 2014a). In the terahertz wavelength regime, photonic crystals are fabricated with length scales of millimeters and are not suitable for silicon-based photonic integrated circuits designed for the IR wavelengths.

Optical resonance sensors based on photonic crystals have several advantages. Photonic crystal cavities typically have high quality factors and very small dimensions, which makes them a promising building block for integrated lab-on-a-chip systems. In a typical photonic crystal cavity, the optical field is mostly located in the high index material, making it difficult to probe the properties of the analyte found within the hollow part of the cavity. To overcome this drawback, we use an air nanocavity in the defect region of a 1-D photonic crystal.

In this paper, a 1-D photonic crystal nanocavity is investigated for refractive index sensing of gases at an IR wavelength of $1.55 \mu \mathrm{m}$. In the photonic crystal, $\mathrm{Si}$ and air layers are used in order to achieve high contrast between the refractive index of the high index and low index adjacent layers and to have a strong optical field in the free space region of the air nanocavity. We show that a high sensitivity of more than $1200 \mathrm{~nm} \mathrm{RIU}^{-1}$ can be obtained for refractive index sensing of gases in the IR. This sensitivity is twice the sensitivity obtained by a 2-D photonic crystal cavity sensor (Jágerská et al., 2010).

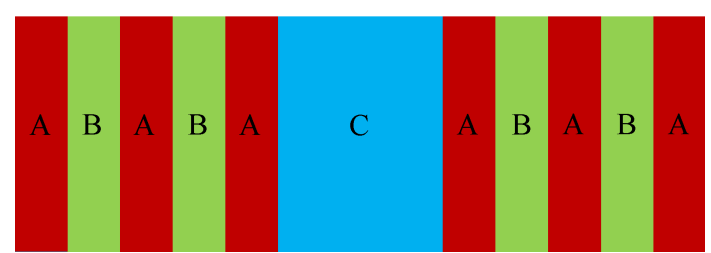

Figure 1. 1-D photonic crystal nanocavity.

\section{Simulation method}

One of the methods used for analysis of photonic crystal structures is the transfer matrix method. We use it to investigate the transmission of light in a 1-D photonic crystal nanocavity as shown in Fig. 1. The electric and magnetic fields inside the layers are shown by $E_{\mathrm{m}}$ and $H_{\mathrm{m}}$. We can then specify the electric and magnetic fields in air, $E_{0}$ and $H_{0}$, by solving the following matrix equation:

$\left[\begin{array}{c}E_{0} \\ H_{0}\end{array}\right]=\mathbf{M}_{1}, \mathbf{M}_{2}, \cdots, \mathbf{M}_{N}\left[\begin{array}{c}E_{\mathrm{m}} \\ H_{\mathrm{m}}\end{array}\right]$,

where $N$ is the total number of layers including the cavity layer and $\mathbf{M}_{j}$ is the characteristic matrix of layer $j$ specified by

$\mathbf{M}_{j}=\left[\begin{array}{cc}\cos \delta_{j} & \left(\frac{-i}{\eta_{j}}\right) \sin \delta_{j} \\ -i \eta_{j} \sin \delta_{j} & \cos \delta_{j}\end{array}\right]$

and

$\delta_{j}=\frac{2 \pi}{\lambda} n_{j} d_{j} \cos \theta_{j}$,

where $\lambda$ is the wavelength, $n_{j}$ is the layer refractive index, $d_{j}$ is the layer thickness, and $\theta_{j}$ is the light's refraction angle in the layer $j$. The parameter $\eta_{j}$ for layer $j$ is defined by

$\eta_{j}=\sqrt{\frac{\varepsilon_{0}}{\mu_{0}}} n_{j} \cos \theta_{j} \quad$ (TE mode)

and

$\eta_{j}=\sqrt{\frac{\varepsilon_{0}}{\mu_{0}}} \frac{n_{j}}{\cos \theta_{j}} \quad$ (TM mode),

where $\varepsilon_{0}$ and $\mu_{0}$ are the permittivity and the permeability of free space, respectively. The transfer matrix of the whole photonic crystal nanocavity is given by

$\mathbf{M}=\mathbf{M}_{1}, \mathbf{M}_{2}, \cdots, \mathbf{M}_{N}=\left[\begin{array}{ll}m_{11} & m_{12} \\ m_{21} & m_{22}\end{array}\right]$.

We can now write the reflection and transmission coefficients of the one-dimensional photonic crystal nanocavity as

$r=\frac{\eta_{0}\left(m_{11}+m_{12} \eta_{\mathrm{m}}\right)-\left(m_{21}+m_{22} \eta_{\mathrm{m}}\right)}{\eta_{0}\left(m_{11}+m_{12} \eta_{\mathrm{m}}\right)+\left(m_{21}+m_{22} \eta_{\mathrm{m}}\right)}$ 
and

$t=\frac{2 \eta_{0}}{\eta_{0}\left(m_{11}+m_{12} \eta_{\mathrm{m}}\right)+\left(m_{21}+m_{22} \eta_{\mathrm{m}}\right)}$,

where $\eta_{0}$ and $\eta_{\mathrm{m}}$ are the parameters for the air and output layers, respectively (as defined by Eqs. 4 or 5). Therefore, the reflectivity $R$ and the transmission $T$ of the whole photonic crystal nanocavity is given by

$R=|r|^{2}$

and

$T=\frac{\eta_{\mathrm{m}}}{\eta_{0}}|t|^{2}$.

\section{Sensor design}

The 1-D photonic crystal nanocavity, shown in Fig. 1, consists of an air nanocavity (layer C) that is introduced as a defect in a 1-D photonic crystal consisting of silicon (A) and air (B) layers. Silicon and air layers are used in order to achieve high contrast of the refractive index between the high index and the low index adjacent layers. To study this structure for gas sensing applications, one would place the structure in a gas cell that has an inlet for injection of gas into the cell and an outlet for gas evacuation. Then, an optical signal from a laser diode (for example, in the wavelength range of 1.4$1.7 \mu \mathrm{m})$ is coupled through a lensed fiber into the photonic crystal cavity. Therefore, the coupling loss is minimized and the transmitted signal would be detectable. The light emitted from the cavity is collected by a microscope objective, which is then detected with a photodiode.

First, the gap map method is used to calculate the photonic crystal band gap. The band gaps as a function of the thickness ratio of air and silicon layers, $d_{\mathrm{Air}} / d_{\mathrm{Si}}$, are shown in Fig. 2. We see that there are different choices of band gaps and thickness ratios for a desired operational wavelength. Four band gap regions labeled by letters $a, b, c$ and $d$ are shown in Fig. 2, which are used in the following analysis.

The 1-D photonic crystal air nanocavity is simulated using the transfer matrix method. The method was explained in the last section in detail, is used to calculate the reflection and transmission properties of the cavity, and gives the amplitude of waves reflected by and transmitted through the cavity. When we choose the thickness of the Si layer $d_{\mathrm{Si}}=0.52 \mu \mathrm{m}$, the thickness of the air layer $d_{\text {Air }}=0.42 \mu \mathrm{m}$, and the cavity length $d_{\text {cavity }}=0.85 \mu \mathrm{m}$, only one cavity mode is excited at a wavelength of $1.55 \mu \mathrm{m}$. The refractive index of the cavity is $n_{\text {cavity }}=n_{\text {Air }}=1.000265$. The transmission of the cavity is shown in Fig. 3, for different cavity lengths. By changing the cavity length, the wavelength of the cavity mode can be adjusted in the band gap $a$ (shown in Fig. 2) from 1.3 to $1.7 \mu \mathrm{m}$.

The cavity wavelength and quality factor have been plotted in Fig. 4, as a function of the cavity length. The wavelength

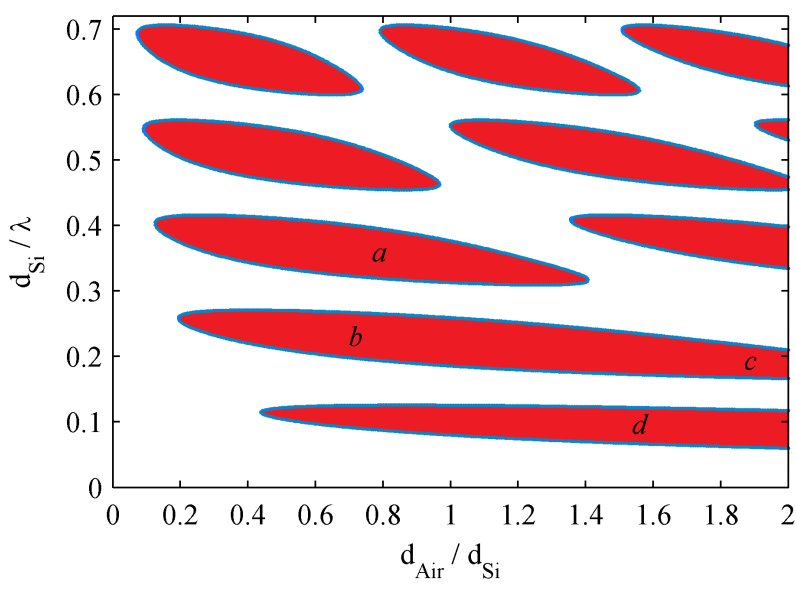

Figure 2. Band gap map of 1-D photonic crystal with silicon and air layers.

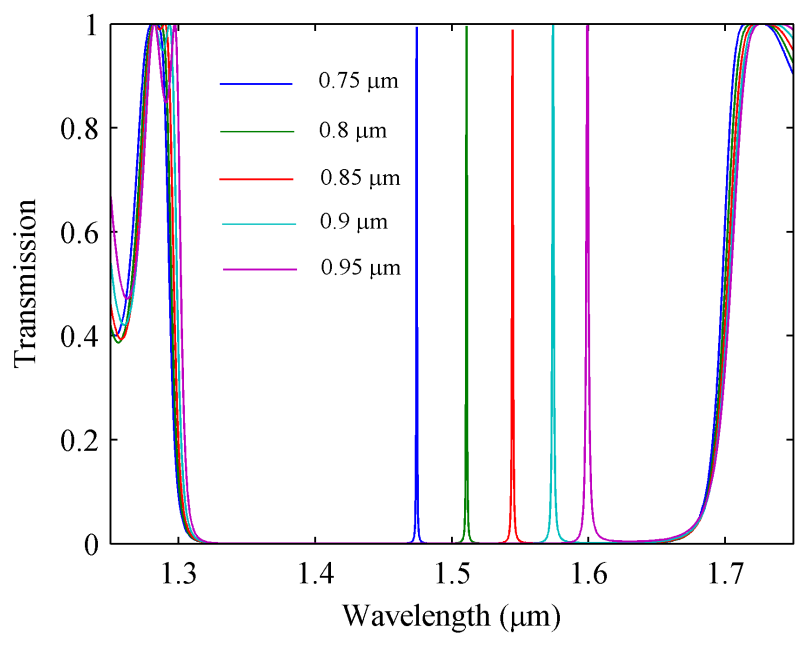

Figure 3. Transmission of the 1-D photonic crystal air nanocavity for $d_{\mathrm{Si}}=0.52 \mu \mathrm{m}$ and $d_{\text {Air }}=0.42 \mu \mathrm{m}$. Different modes oscillate for cavity lengths of $0.75-0.95 \mu \mathrm{m}$ (from left to right).

of the defect mode shifts to higher wavelengths almost linearly, when the cavity length is increased. The quality factor decreases as the wavelength shifts to the edges of the band gap, which results in a broadened bandwidth, and peaks at a wavelength near the middle of the band gap. For sensing applications, it is desirable to have a mode with a narrow bandwidth and, thus, we must choose the parameters so that the quality factor peaks at the operation wavelength. One can see that the cavity has a high quality factor on the order of $10^{6}$, which results in an optical sensor with high sensitivity.

To study the photonic crystal air nanocavity for refractive index sensing of gases, we consider gases of different refractive indices, such as air $(n=1.000265), \mathrm{N}_{2}(n=1.000270)$, $\mathrm{He}(n=1.000032)$, and $\mathrm{CO}_{2}(n=1.000407)$. The cavity supports a single defect mode, which is shifted in the band gap when the refractive index of the gas is changed. The 


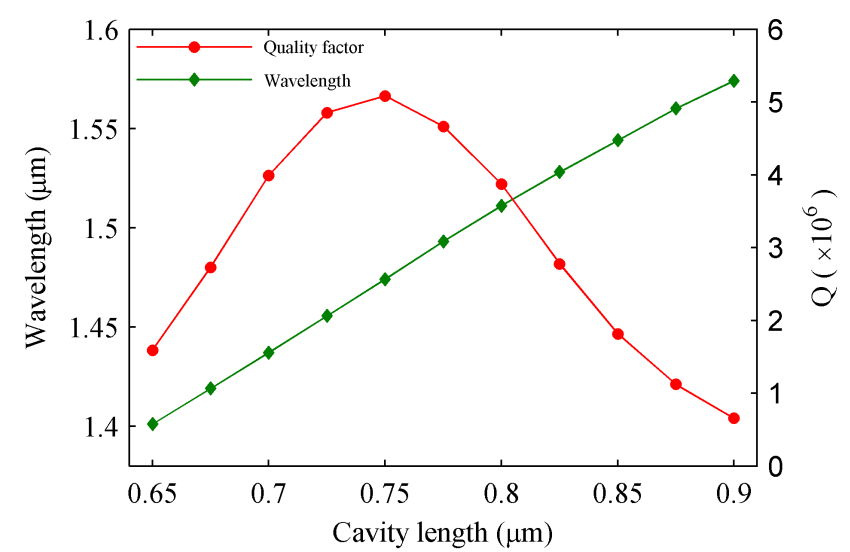

Figure 4. The cavity wavelength and quality factor as a function of cavity length for $d_{\mathrm{Si}}=0.52 \mu \mathrm{m}$ and $d_{\text {Air }}=0.42 \mu \mathrm{m}$.

transmission and spectral position of the cavity mode for different gases are shown in Fig. 5. Air has been used as the reference medium. When a different gas is considered, both blue shift (for $\mathrm{He}$ ) and red shift (for $\mathrm{CO}_{2}$ ) of the wavelength of the cavity mode occur. In Fig. 5, the transmission of the cavity mode has been shown for four periodic layers on each side of the cavity. The full width at half maximum (FWHM) bandwidth of the cavity mode is $4.3 \mathrm{pm}$. Therefore, by using a small number of periodic layers in the structure, it is possible to obtain very narrow bandwidths. The ratio of the FWHM bandwidth to the wavelength shift (for $\mathrm{CO}_{2}$ ) is calculated to be 0.05 , indicating that the sensor has a very good resolution.

The sensitivity of the sensor is defined by $S=\Delta \lambda / \Delta n$, where $\Delta \lambda$ is the wavelength shift of the cavity mode and $\Delta n$ is the refractive index change of the gas with respect to the air refractive index. Figure 6 shows the calculated sensitivities for four air-slot nanocavities with different $d_{\mathrm{Si}}$ and $d_{\text {Air }}$ as a function of the cavity length. The cavities operate in different band gap regions corresponding to the band gaps labeled by letters $a, b, c$, and $d$ as shown in Fig. 2. The central wavelength of the cavity mode changes in the IR range, as the cavity length is changed. By choosing the parameters, one can design the cavity for a desired operational wavelength. The sensitivity curves exhibit a peak at a specific cavity length, which depends on the values of $d_{\mathrm{Si}}$ and $d_{\mathrm{Air}}$. It is clearly seen that, as the length of the silicon layer $d_{\mathrm{Si}}$ gets smaller, the sensitivity becomes larger.

To study the effect of the air layer on the sensitivity of the optical sensor, we selected $d_{\mathrm{Si}}=0.32 \mu \mathrm{m}$, and calculated the sensitivity as a function of the air layer length $d_{\text {Air }}$. The length of the cavity was changed, from 0.725 to $0.825 \mu \mathrm{m}$, in order to keep the central wavelength of the cavity mode at $1.55 \mu \mathrm{m}$ as the length of the air layer was changed. As shown in Fig. 7, the sensitivity is almost linearly related to $d_{\text {Air }}$ and increases as the length of the air layer is increased.

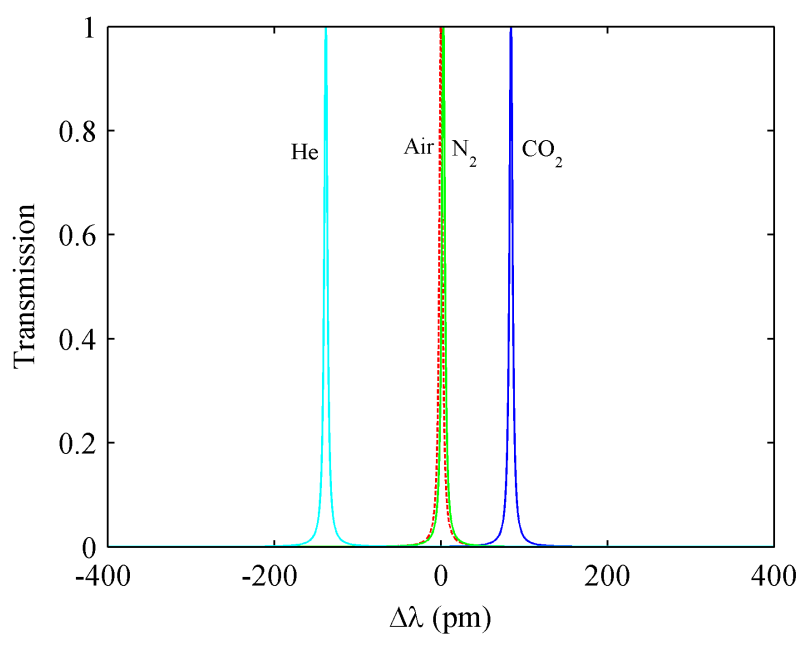

Figure 5. Transmission and spectral position of the cavity mode for different gases with air as the reference medium. The mode has a very narrow bandwidth of $4.3 \mathrm{pm}$.

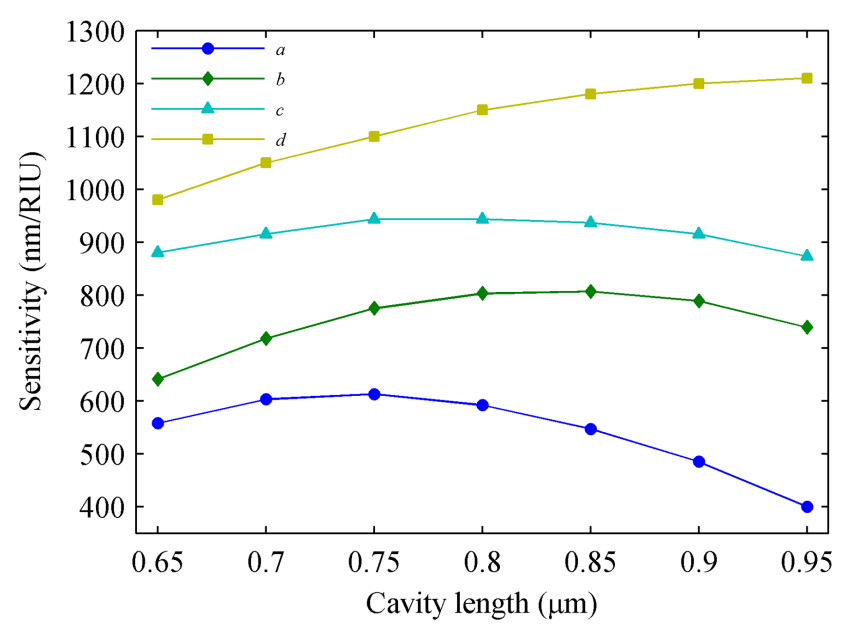

Figure 6. Sensitivity for different values of $d_{\mathrm{Si}}$ and $d_{\mathrm{Air}}$ as a function of the cavity length. The cavities operate in different band gap regions shown in Fig. 2: $a\left(d_{\mathrm{Si}}=0.52 \mu \mathrm{m}\right.$ and $\left.d_{\text {Air }}=0.42 \mu \mathrm{m}\right), b$ $\left(d_{\mathrm{Si}}=0.36 \mu \mathrm{m}\right.$ and $\left.d_{\mathrm{Air}}=0.26 \mu \mathrm{m}\right), c\left(d_{\mathrm{Si}}=0.32 \mu \mathrm{m}\right.$ and $d_{\mathrm{Air}}=$ $0.6 \mu \mathrm{m})$, and $d\left(d_{\mathrm{Si}}=0.14 \mu \mathrm{m}\right.$ and $\left.d_{\mathrm{Air}}=0.22 \mu \mathrm{m}\right)$.

The shift in the cavity mode wavelength $\Delta \lambda$ in response to a change in the gas refractive index with respect to the air refractive index $\Delta n$ is shown in Fig. 8. The results are for four cavities with different $d_{\mathrm{Si}}$ and $d_{\mathrm{Air}}$ as used in Fig. 6, corresponding to band gaps $a, b, c$, and $d$. The cavity length has been chosen so that all cavities operate at a central wavelength of $1.55 \mu \mathrm{m}$. It is seen that the wavelength shift is related linearly to the refractive index change and is higher for smaller values of the silicon layer thickness, reaching a value of $1106 \mathrm{~nm} \mathrm{RIU}^{-1}$ for $d_{\mathrm{Si}}=0.14 \mu \mathrm{m}, d_{\mathrm{Air}}=0.22 \mu \mathrm{m}$, and $d_{\text {cavity }}=0.75 \mu \mathrm{m}$. The sensitivity of the 1-D photonic crystal nanocavity sensor analyzed here is higher than (almost twice) the sensitivity of 2-D photonic crystal cavity sensors 


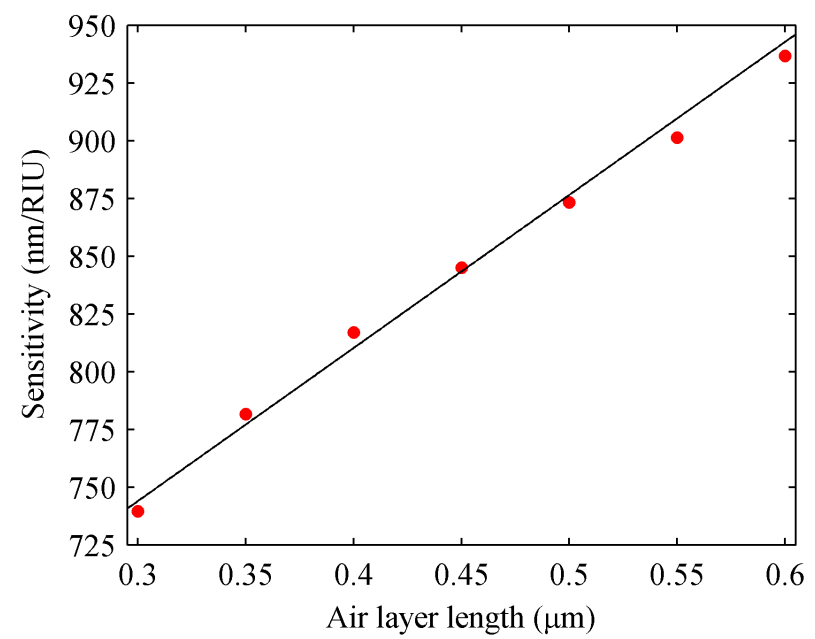

Figure 7. Sensitivity as a function of the air layer length for $d_{\mathrm{Si}}=0.32 \mu \mathrm{m}$. The cavity length is changed from 0.725 to $0.825 \mu \mathrm{m}$ in order to keep the cavity wavelength at $1.55 \mu \mathrm{m}$. The sensitivity changes almost linearly with $d_{\text {Air }}$.

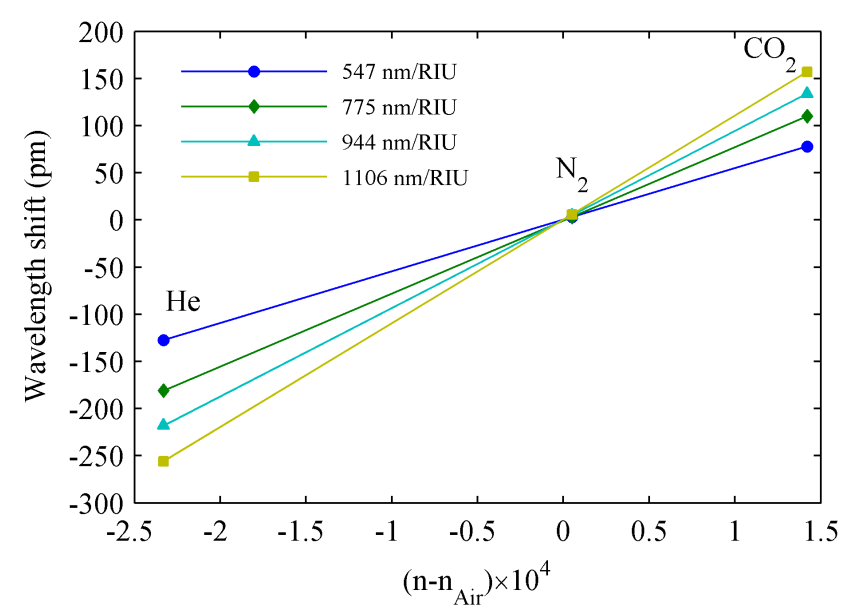

Figure 8. The wavelength shift as a function of the refractive index. The results are for cavities corresponding to band gaps $a$ (circle), $b$ (diamond), $c$ (triangle), and $d$ (square). The cavity length has been adjusted so that all cavities operate at a central wavelength of $1.55 \mu \mathrm{m}, d_{\text {cavity }}=0.85 \mu \mathrm{m}$ for $a$ and $d_{\text {cavity }}=0.75 \mu \mathrm{m}$ for $b, c$, and $d$.

reported previously. In addition, it has a simpler design and can be easily fabricated as part of silicon-based photonic integrated circuits designed for operation in the near-IR wavelength range.

\section{Optimization of the sensor}

In this section, we present a method for optimizing the sensor sensitivity. In sensing applications, a sensor is required to have high sensitivity and good resolution. We showed above that the sensitivity is increased as the air layer is made thicker. However, in optical resonance sensors, the FWHM

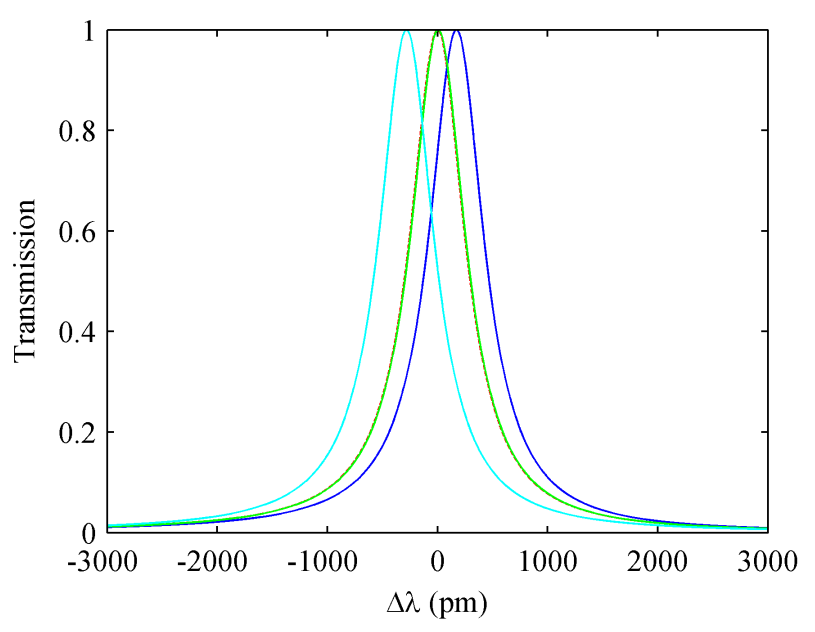

Figure 9. Transmission and spectral position of the cavity mode for different gases, with $d_{\mathrm{Si}}=0.14 \mu \mathrm{m}, d_{\text {Air }}=0.55 \mu \mathrm{m}$, and $d_{\text {cavity }}=$ $0.645 \mu \mathrm{m}$. The mode has a very broad bandwidth, yielding a sensor with poor resolution.

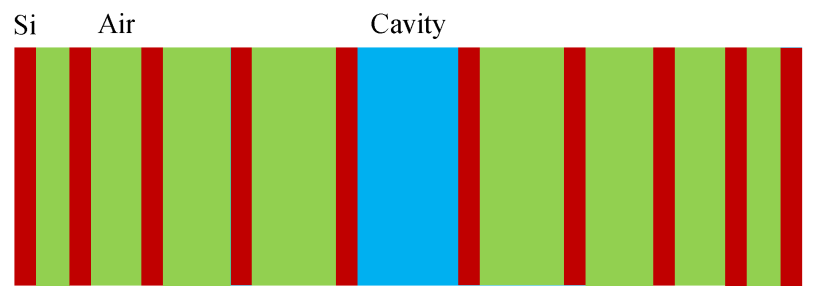

Figure 10. 1-D photonic crystal nanocavity with the thickness of the air layers changing linearly. The thickness of the air layer is increased linearly from 0.22 to $0.55 \mu \mathrm{m}$ on the left side of the cavity and is decreased linearly from 0.55 to $0.22 \mu \mathrm{m}$ on the right side of the cavity. The cavity length is $d_{\text {cavity }}=0.66 \mu \mathrm{m}$ for a cavity mode with a central wavelength of $1.55 \mu \mathrm{m}$.

bandwidth of the cavity mode is very important and affects the sensor resolution. If the bandwidth is broad, detecting a small change in the gas refractive index becomes difficult. In order to investigate this problem, we consider the sensor with $d_{\mathrm{Si}}=0.14 \mu \mathrm{m}$ and $d_{\mathrm{Air}}=0.22 \mu \mathrm{m}$. First, to obtain a higher sensitivity, we increase the thickness of the air layer to $d_{\text {Air }}=0.55 \mu \mathrm{m}$. The cavity length is changed to $d_{\text {cavity }}=0.645 \mu \mathrm{m}$ in order to have a cavity mode with a central wavelength of $1.55 \mu \mathrm{m}$. The number of periodic layers in the photonic crystals has not been changed and is four. The transmission and spectral position of the cavity mode are shown in Fig. 9 for the same gases as before. There is a higher shift of $1200 \mathrm{~nm} \mathrm{RIU}^{-1}$ in the position of the mode peak as compared to the sensor with $d_{\text {Air }}=0.22 \mu \mathrm{m}$. However, as can be seen from Fig. 9, the cavity mode has a very broad bandwidth, which makes the detection of the gas difficult.

Now, we propose a method by which the thickness of the air layers in the 1-D photonic crystals are changed linearly. 


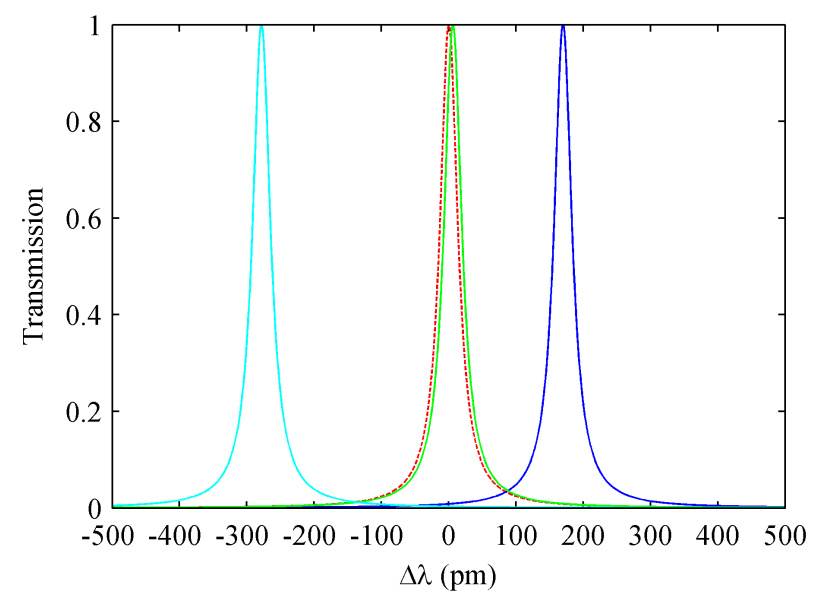

Figure 11. Transmission and spectral position of the cavity mode for the 1-D photonic crystal nanocavity with linearly changing air layer thickness as shown in Fig. 10. The mode has a very narrow bandwidth, yielding a sensor with good resolution.

We change the air layer thickness in each photonic crystal linearly by a constant value. A 1-D photonic crystal nanocavity with linearly changing air layer thicknesses is shown in Fig. 10. The thickness of the air layer in the photonic crystal on the left side of the cavity is increased as

$\left(d_{\text {Air }}\right)_{j}=\left(d_{\text {Air }}\right)_{1}+(j-1)(\delta B), \quad j=1,2, \ldots, n$,

where $\left(d_{\text {Air }}\right)_{1}=0.22 \mu \mathrm{m}$ is the thickness of the first air layer, $\delta B=0.11 \mu \mathrm{m}$ is the amount that is added to the air layer thickness in each step, and $n=4$ is the number of periodic layers in the photonic crystal. In this example, the amount of change in the air layer thickness is chosen so that the thickness of the last air layer becomes $0.55 \mu \mathrm{m}$.

The thickness of the air layer in the photonic crystal on the right side of the cavity is decreased as

$\left(d_{\text {Air }}\right)_{j}=\left(d_{\text {Air }}\right)_{1}+(n-j)(\delta B), \quad j=1,2, \ldots, n$,

where the values of different parameters are as before. Therefore, the thickness of the air layer is increased linearly from 0.22 to $0.55 \mu \mathrm{m}$ in the photonic crystal on the left side of the cavity and is decreased linearly from 0.55 to $0.22 \mu \mathrm{m}$ in the photonic crystal on the right side of the cavity. The cavity length is $d_{\text {cavity }}=0.66 \mu \mathrm{m}$ in order to have a cavity mode with a central wavelength of $1.55 \mu \mathrm{m}$. The transmission and spectral position of the cavity mode for this new design are shown in Fig. 11. As can be seen, in this case, the cavity mode has a very narrow bandwidth compared to the mode shown in Fig. 9 for the case where the air layer has a constant value of $0.55 \mu \mathrm{m}$. The sensitivity of this sensor is shown in Fig. 12 and is $1200 \mathrm{~nm} \mathrm{RIU}^{-1}$. Therefore, by using this method, we are able to optimize the sensor such that it has a higher sensitivity and a very narrow cavity mode for good sensor resolution.

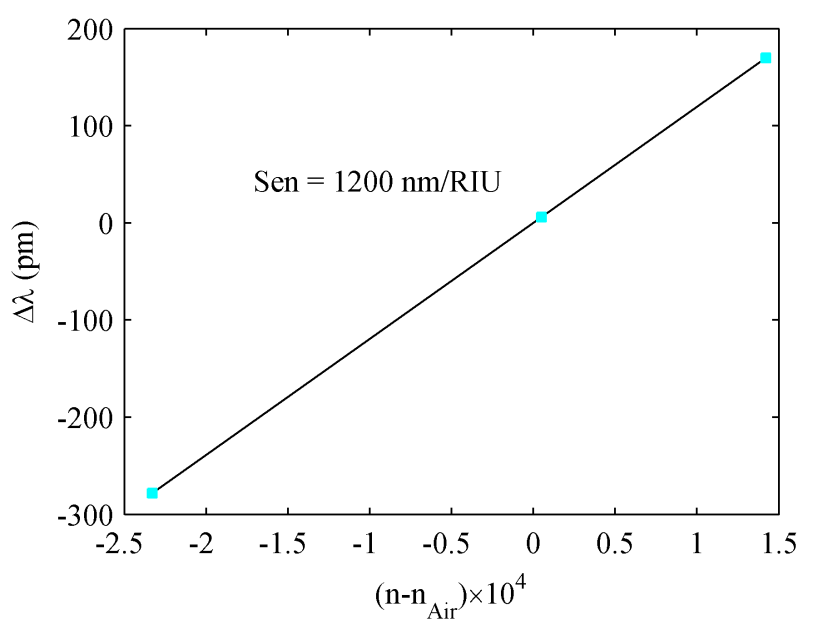

Figure 12. Sensitivity of the optimized sensor that has the structure shown in Fig. 10.

\section{Conclusions}

A 1-D photonic crystal nanocavity was investigated for refractive index sensing of gases at a wavelength of $1.55 \mu \mathrm{m}$. An air defect is used to create a cavity in the photonic crystal. The sensor monitors the resonance shift of the cavity as a function of the refractive index of the gas found within the cavity. It was seen that a smaller thickness of the high index material (Si) results in higher sensitivity. Larger thickness of the low index material (air) also increases the sensitivity of the sensor. A method was presented in order to optimize the sensitivity and resolution of the sensor. By linearly changing the thicknesses of the air layers in the photonic crystals on either side of the nanocavity, we are able to obtain a high sensitivity of $1200 \mathrm{~nm} \mathrm{RIU}^{-1}$ and a very narrow bandwidth cavity mode for good sensor resolution. The sensor presented here has a simple structure and can be integrated into silicon-based photonic circuits.

Edited by: M. J. da Silva

Reviewed by: two anonymous referees

\section{References}

Bougriou, F., Bouchemat, T., Bouchemat, M., and Paraire, N.: Optofluidic sensor using two-dimensional photonic crystal waveguides, Eur. Phys. J. Appl. Phys., 62, 11201-11205, 2013.

Chen, T., Han, Z., Liu, J., and Hong, Z.: Terahertz gas sensing based on a simple one-dimensional photonic crystal cavity with highquality factors, Appl. Opt., 53, 3454-3458, 2014a.

Chen, T., Liu, P., Liu, J., and Hong, Z.: A terahertz photonic crystal cavity with high Q-factors, Appl. Phys. B, 115, 105-109, 2014b.

Fang, X., Liao, C. R., and Wang, D. N.: Femtosecond laser fabricated fiber Bragg grating in microfiber for refractive index sensing, Opt. Lett., 35, 1007-1009, 2010. 
Frascella, F., Ricciardi, S., Rivolo, P., Moi, V., Giorgis, F., Descrovi, E., Michelotti, F., Munzert, P., Danz, N., Napione, L., Alvaro, M., and Bussolino, F.: A fluorescent One-dimensional photonic crystal for label-free biosensing based on Bloch surface waves, Sensors, 13, 2011-2022, 2013.

Jágerská, J., Zhang, H., Diao, Z., Thomas, N., and Houdré, R.: Refractive index sensing with an air-slot photonic crystal nanocavity, Opt. Lett., 35, 2523-2525, 2010.

Kang, C., Phare, C. T., Vlasov, Y. A., Assefa, S., and Weiss, S. M.: Photonic crystal slab sensor with enhanced surface area, Opt. Express, 18, 27930-27937, 2010.

Lee, H. W., Schmidt, M. A., Uebel, P., Tyagi, H., Joly, N. Y., Scharrer, M., and Russell, P. S. J.: Optofluidic refractive index sensor in step-index fiber with parallel hollow micro-channel, Opt. Express, 19, 8200-8207, 2011.

Liu, Y. and Salemink, H. W. M.: Photonic crystal based all optical on chip sensor, Opt. Express, 20, 19912-19920, 2012.
Ma, L., Katagiri, T., and Matsuura, Y.: Surface-plasmon resonance sensor using silica-core Bragg fiber, Opt. Lett., 34, 1069-1071, 2009.

Nunes, P. S., Mortensen, N. A., Kutter, J. P., and Mogensen, K. B.: Refractive index sensor based on a 1-D photonic crystal in a microfluidic channel, Sensors, 10, 2348-2358, 2010.

Sreekantha, K. V., Zeng, S., Yongb, K., and Yu, T.: Sensitivity enhanced biosensor using graphene-based one-dimensional photonic crystal, Sensor. Actuat. B.-Chem., 182, 424- 428, 2013.

Udd, E. and Spillman, W.: Fiber Optic Sensors - an Introduction for Engineers and Scientists, Wiley, Hoboken, New Jersey, USA, 2011.

Vangala, S. R., Avrutsky, I., Keiffer, P., Nader, N., Walker Jr, D., Cleary, J. W., and Hendrickson, J. R.: Asymmetric photonic resonances in $\mathrm{GaN}$ slab waveguide for mid infrared selective filters, Opt. Express, 22, 24742-24751, 2014. 